\title{
Obstrucción duodenal por Strongyloides stercoralis, una complicación inusual
}

\author{
Fernando Soto-Febres', Giancarlo Pérez-Lazo', Walter Anicama² y Julio Maquera-Afaray3,4
}

\section{Duodenal obstruction by Strongyloides stercoralis, an unusual complication}

Strongyloidiasis is a neglected disease in Latin America. Gastrointestinal manifestations are nonspecific and duodenal obstruction is a rare complication. Here we present the case of a 31-year-old male from the central jungle of Peru, admitted due to a high intestinal obstruction, with duodenal ulcers and stenosis evidenced in the upper endoscopy. The histopathological report revealed presence of larvae of Strongyloides stercoralis. Clinical and endoscopic follow up were favorable with ivermectin treatment. There are near 20 reported cases of duodenal obstruction due to $S$. stercoralis. Additionally, infection by HTLV-1 was confirmed, being this a frequent association.

Keywords: Duodenal obstruction; Strongyloides; HTLV-1.

Palabras clave: Obstrucción duodenal; Strongyloides; HTLV-1.

\section{Introducción}

\section{$\mathrm{L}$}

a estrongiloidosis es una parasitosis producida por el nemátodo Strongyloides stercoralis ${ }^{1}$ y la prevalencia global es poco conocida debido a la falta de estudios representativos en diversos países, por tratarse de una enfermedad desatendida ${ }^{2}$.

Las manifestaciones gastrointestinales son inespecíficas y variadas, desde alteraciones de la frecuencia defecatoria, dolor abdominal epigástrico y síndrome de intestino irritable. Sin embargo, la obstrucción intestinal por $S$. stercoralis es una complicación con alta mortalidad, muy infrecuente y pobremente reconocida ${ }^{3,4}$. Presentamos el caso de un adulto con una obstrucción duodenal causada por $S$. stercoralis asociada a una infección por HTLV-1.

\section{Caso clínico}

Varón de 31 años, natural de la selva central de Perú (distrito de Pichanaqui - región Junín), sin antecedentes médicos de importancia. Acudió al servicio de urgencia por tres semanas de fiebre, distensión abdominal, diarrea (dos a tres episodios al día) y pérdida de peso de aproximadamente $5 \mathrm{~kg}$. Una semana después se agregaron síntomas compatibles con una obstrucción intestinal alta. Al examen físico del ingreso destacaba un paciente adelgazado, afebril, con presión arterial: 90/60 mmHg y FC: 126 latidos por minuto. Presentaba dolor a la palpación en hemiabdomen superior, sin signos de irritación peritoneal.

Los exámenes de ingreso evidenciaron una leucocitosis con desviación izquierda (14.240 céls $/ \mathrm{mm}^{3}$, con $20 \%$ baciliformes sin eosinófilos, hemoglobina: 14,3 g/dl, hipoalbuminemia $(2,1 \mathrm{~g} / \mathrm{dl})$ e hiponatremia (117 meq/l). Se corrigió la hiponatremia y se inició terapia antibacteriana empírica con ciprofloxacina iv $400 \mathrm{mg}$ fraccionado cada $12 \mathrm{~h}$, asociado a metronidazol iv $500 \mathrm{mg}$ cada $8 \mathrm{~h}$ por posible sepsis de foco intraabdominal. La tomografía computada (TC) abdominal con contraste evidenció una distensión gástrica y engrosamiento mural con realce mucoso en asas del intestino delgado y grueso, sin definir lesiones focales estenosantes (Figura 1). Se realizó una primera endoscopia, la cual no se completó por marcada retención de residuos alimentarios. Se realizó una nueva endoscopia dos días después, evidenciándose una mucosa gástrica eritematosa, y antro de distensibilidad disminuida; píloro entreabierto de aspecto rígido, con pliegues eritematosos; bulbo y segunda porción duodenal con mucosa ulcerada con extensas áreas cubiertas de fibrina, focos de granulación y friabilidad al roce, que comprometían toda la circunferencia produciendo estenosis e imposibilitando el progreso del duodenoscopio. Se tomaron biopsias de la región antro-pilórica y duodenal. A pesar de cinco días de cobertura antibacteriana persistió con fiebre, por lo que se cambió a imipenem iv $500 \mathrm{mg}$ cada $6 \mathrm{~h}$.

Al décimo día de hospitalización cursó con una hemorragia digestiva alta con repercusión hemodinámica y disminución de hemoglobina a 10,5 g/dl, tratadas con expansores de volumen y transfusión de glóbulos rojos. En el aspirado gástrico se evidenció la presencia de abundantes larvas rabditiformes de $S$. stercoralis. Recibió tratamiento de tres ciclos semanales de ivermectina $200 \mu \mathrm{g} / \mathrm{kg}$, por dos días consecutivos administrados por SNG, con controles negativos de parásitos en aspirado
'Unidad de Infectología, Hospital Nacional Guillermo Almenara Irigoyen - EsSALUD. Lima, Perú. ${ }^{2}$ Servicio de Anatomía Patológica Hospital Nacional Guillermo Almenara Irigoyen - EsSALUD. Lima, Perú.

${ }^{3}$ Instituto Nacional de Salud del Niño San Borja. Lima, Perú. ${ }^{4}$ Escuela de Medicina, Universidad Privada de Tacna. Tacna, Perú.

Los autores declaran no tener conflictos de interés. Sin financiamiento.

Recibido: 21 de diciembre de 2017

Aceptado: 3 de diciembre de 2018

Correspondencia a: Fernando Soto-Febres fernando.soto.f@upch.pe 


\section{Caso Clínico}

Figura 1. Tomografía computada abdominal (TC) con contraste. Estómago parcialmente distendido, engrosamiento mural con realce mucoso en asas intestinales delgadas y gruesas (flechas), sugerente de una enterocolitis.
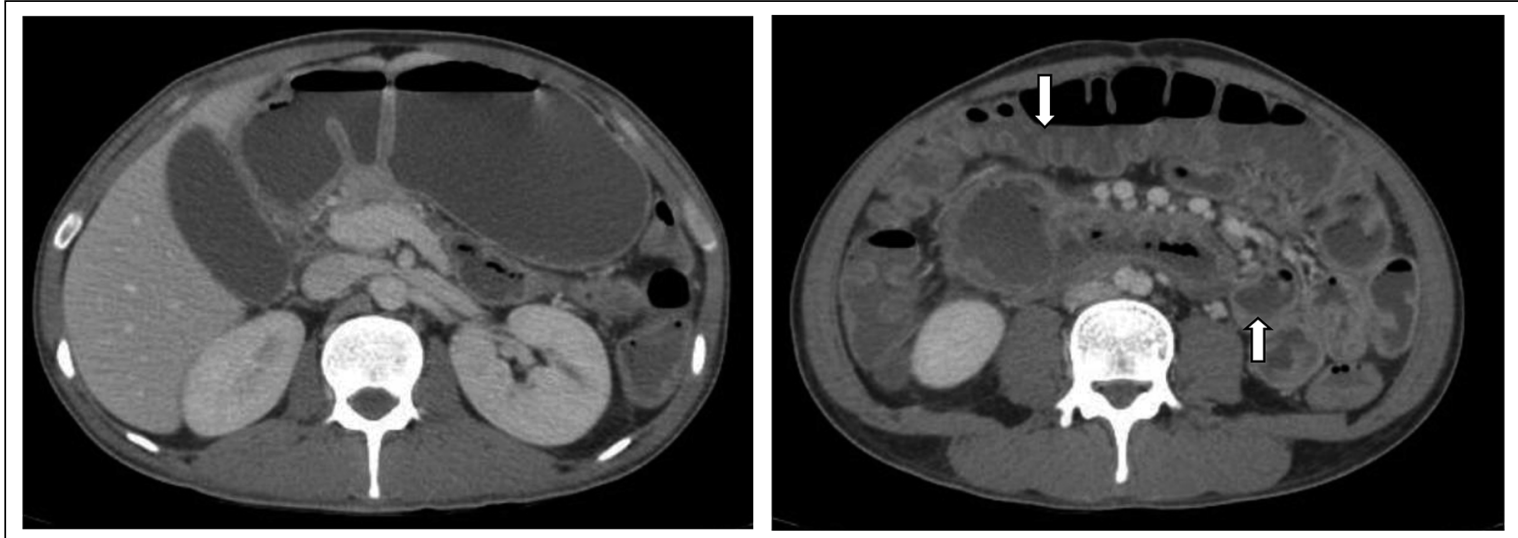

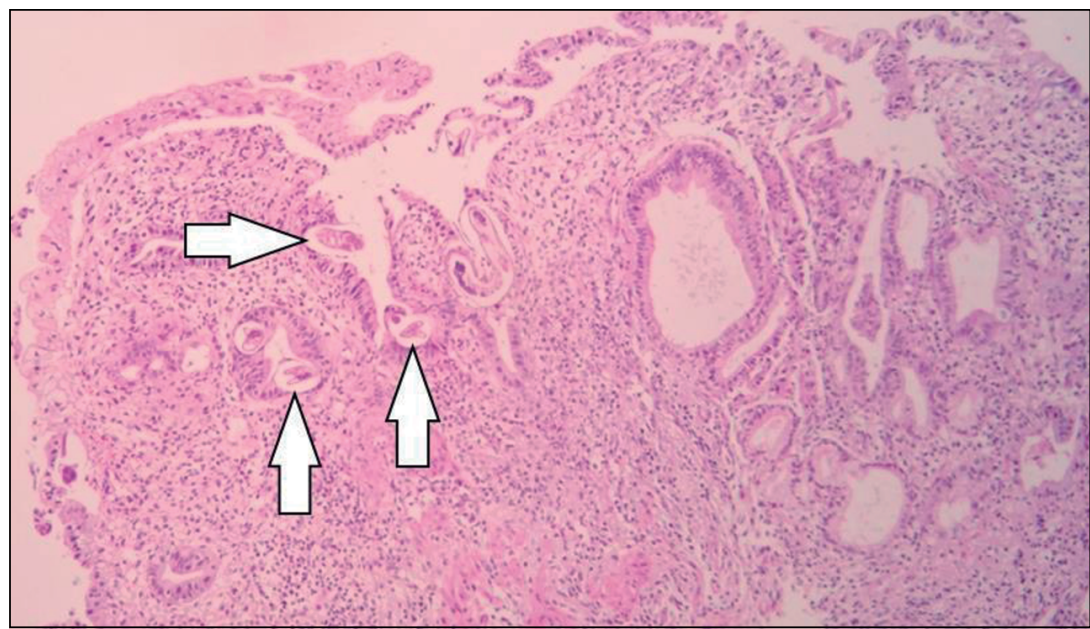

Figura 2. Mucosa gástrica con presencia de huevos con larvas y larvas rabditiformes libres (flechas). Tinción de hematoxilina y eosina (10x).

Figura 3. Presencia de hembras adultas de Strongyloides stercoralis (flecha grande), obsérvese la presencia de los ovarios en un corte transversal de una hembra adulta (flecha pequeña). Tinción de hematoxilina y eosina (40x).

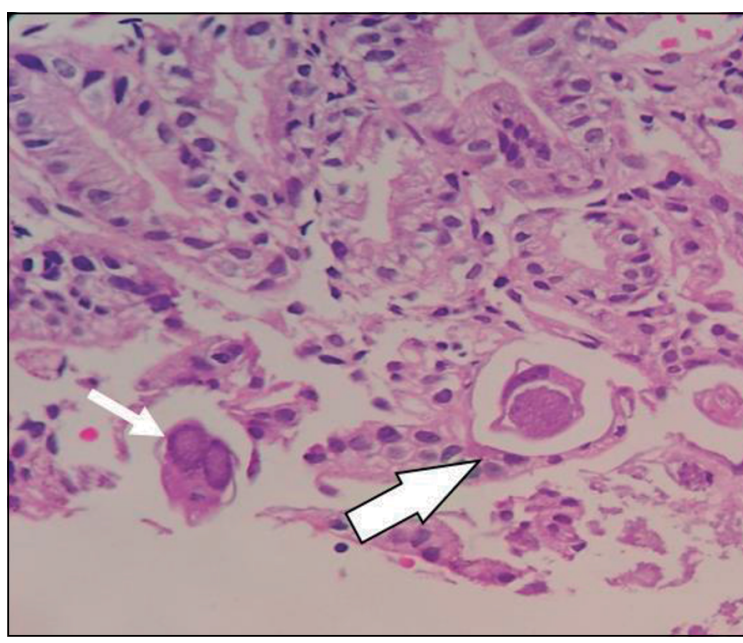

gástrico luego del primer ciclo. El estudio de parásitos en esputo fue negativo al finalizar cada ciclo semanal. No se obtuvieron muestras para búsqueda de parásitos en heces debido a la obstrucción intestinal. Se decidió solicitar serología para HTLV-1 que resultó reactiva (método quimioluminiscencia-ECLIA).

El estudio histopatológico de las biopsias endoscópicas reveló un nicho ulceroso con inflamación intensa y reacción gigantocelular a cuerpo extraño en el estómago y duodeno, con presencia de larvas y formas adultas de $S$. stercoralis (Figuras 2 y 3). Completó tratamiento con imipenem por 14 días, evidenciándose defervescencia y disminución de los biomarcadores inflamatorios. Además, recibió nutrición parenteral total por ocho días, con un reinicio posterior de nutrición por vía oral. Se realizó control de endoscopia a los 20 días, donde se evidenciaron escasas erosiones pequeñas en región pre-pilórica, con mucosa del bulbo duodenal de aspecto irregular, nodular, friable al roce con extensión hacia la segunda porción, produciendo estenosis hasta $80 \%$ a ese nivel. En el seguimiento del paciente, al mes del alta hospitalaria, se observó una ganancia ponderal y controles parasitológicos en heces (por métodos de concentración y cultivo de agar) negativos. En el estudio familiar de HTLV-1 se encontró una prueba reactiva en la madre.

\section{Discusión}

El ciclo de vida de $S$. stercoralis es complejo y puede perpetuarse mediante el ciclo de autoinfección intestinal, caracterizado por la maduración intraluminal y posterior transformación a larvas filariformes, las cuales penetran la piel perianal o mucosa colónica. Puede presentarse de forma asintomática u oligosintomática y bajo ciertas condiciones de inmunosupresión pueden conllevar a un estado de hiperinfección y/o diseminación de la enfermedad ${ }^{1}$. 
Strongyloides stercoralis afecta principalmente las primeras porciones del intestino delgado ${ }^{1}$, provocando manifestaciones clínicas variadas y frecuentes, desde alteraciones de la frecuencia defecatoria, deposiciones acuosas, mucoides e incluso esteatorreicas (que pueden llevar a un síndrome malabsortivo sprue-like), estreñimiento, dolor abdominal epigástrico, distensión abdominal y síndrome de intestino irritable ${ }^{5}$; además, de otros procesos inflamatorios de la mucosa que pueden llegar a producir obstrucción intestinal subaguda, hemorragia digestiva, infarto mesentérico y perforación intestinal ${ }^{1,3,4,6,7}$.

Se han descrito en la literatura médica 23 casos de obstrucción duodenal por S. stercoralis s $^{3,4,6,7-15}$. En la Tabla 1 se resumen ciertas características de los casos encontrados, siendo la mayoría hombres, con una edad promedio de 41 años, y con infección por HTLV-1 como la condición asociada más frecuente. La eosinofilia periférica estuvo ausente en $80 \%$ de los casos, probablemente por las condiciones de inmunosupresión. A su vez, 37\% de los pacientes fueron sometidos a cirugía, de los cuales en dos tercios se realizó una resección intestinal, debido a la obstrucción y/o al compromiso de la viabilidad del asa.

El síndrome de hiperinfección en nuestro caso fue manifestado clínicamente por el compromiso gastrointestinal, incluyendo una enteropatía perdedora de proteínas y diarrea con dolor abdominal, que explican la malnutrición e hipoalbuminemia asociada ${ }^{16}$. También cursó con una sepsis intraabdominal con respuesta apropiada al tratamiento antimicrobiano de amplio espectro. Es importante reconocer la sobreinfección bacteriana como una complicación secundaria a estrongiloidiasis, debido al arrastre de enterobacterias en la cutícula de las larvas o por disrupción de la mucosa intestinal, con complicaciones respiratorias secundarias a la sepsis o la migración directa de las larvas al parénquima pulmonar ${ }^{7}$. En nuestro caso no hubo confirmación microbiológica de sobreinfección.

La eosinofilia es un indicador de infección en poblaciones de riesgo, pero no es suficientemente sensible para ser usada como un marcador de monitoreo único en la infección crónica no complicada debido a su presencia intermitente ${ }^{1,16}$. La mayoría de los pacientes con síndrome de hiperinfección no presentan eosinofilia periférica, y en la coinfección con HTLV-1 se documenta adicionalmente bajos niveles de inmunoglobulina $\mathrm{E}$, importantes en la defensa del hospedero contra el parásito ${ }^{17}$. Entre los casos encontrados de obstrucción duodenal por S. stercoralis, 10 tuvieron coinfección con HTLV-1, y solo uno entre estos presentó eosinofilia moderada.

Aunque el examen directo en deposiciones es un método barato y sencillo, tiene una sensibilidad variable en casos de obstrucción intestinal por $S$. stercoralis; siendo indispensable en estos casos su búsqueda, además, en aspirado gástrico y secreciones bronquiales. Sin embargo, en comparación a las pruebas parasitológicas, la serología
Tabla 1. Características de los casos de obstrucción duodenal por Strongyloides stercoralis publicados, incluido el caso presentado en esta comunicación $(n=24)$

\begin{tabular}{|c|c|}
\hline Característica & $\begin{array}{c}\text { Frecuencia } \\
\text { (\%) }\end{array}$ \\
\hline Promedio de edad en años (rango)* & $41,6(9-70)$ \\
\hline Sexo masculino & $18(75 \%)$ \\
\hline $\begin{array}{l}\text { Comorbilidades } \\
\text { HTLV-1 } \\
\text { Cáncer hematológico } \\
\text { Trasplante de órganos sólidos } \\
\text { Corticoterapia } \\
\text { Sin co-morbilidades }\end{array}$ & $\begin{array}{rr}10 & (41,6 \%) \\
3 & (12,5 \%) \\
2 & (8,3 \%) \\
1 & (4,1 \%) \\
8 & (33,5 \%)\end{array}$ \\
\hline Eosinofilia & $5(20,8 \%)^{*}$ \\
\hline $\begin{array}{l}\text { Cirugía } \\
\text { Resección intestinal } \\
\text { Sin resección intestinal }\end{array}$ & $\begin{array}{l}9(37,5 \%) \\
6(25 \%) \\
3(12,5 \%)\end{array}$ \\
\hline $\begin{array}{l}\text { Diagnóstico } \\
\text { Biopsia endoscópica } \\
\text { Pieza quirúrgica } \\
\text { Deposiciones y secreciones biológicas (lavado broncoalveolar, aspirado gástrico) }\end{array}$ & $\begin{array}{c}15(62,5 \%) \\
4(16,5 \%) \\
5(21 \%)\end{array}$ \\
\hline $\begin{array}{l}\text { Tratamiento antiparasitario } \\
\text { Ivermectina } \\
\text { Tiabendazol } \\
\text { Albendazol } \\
\text { Albendazol + ivermectina }\end{array}$ & $\begin{array}{l}8(33,3 \%) \\
5(20,8 \%) \\
2 \quad(8,3 \%) \\
9(37,6 \%)\end{array}$ \\
\hline Mortalidad & $4(16,6 \%)$ \\
\hline
\end{tabular}

*Tres presentaron eosinofilia moderada (1.500-5.000 céls/mm³) y dos eosinofilia leve (500-1.499 céls $/ \mathrm{mm}^{3}$ ).

tiene mayor sensibilidad (56-100\%, según la técnica), pero con la limitación de no poder distinguir una infección pasada de una actual, menor sensibilidad en viajeros que retornan de zonas endémicas (por menor exposición al parásito) y en personas inmunocompetentes (40\%), por reacciones cruzadas con otros nemátodos ${ }^{18}$.

La biopsia endoscópica constituye un método diagnóstico útil (62\%) en los casos de obstrucción duodenal descritos. Los hallazgos endoscópicos a nivel gástrico y duodenal son una gastroduodenopatía eritematosa petequial asociado a múltiples ulceraciones, similares a nuestro caso.

Los cambios histopatológicos que $S$. stercoralis causa en la mucosa intestinal se clasifican como enteritis catarral, edematosa y ulcerativa según el infiltrado inflamatorio, atrofia de vellosidades y presencia de úlceras. En un estudio realizado en un hospital peruano que incluyeron 11 muestras obtenidas por biopsia duodenal reportaron 
como hallazgos principales la atrofia (vellosidades totalmente planas) y un infiltrado inflamatorio intenso que incluía polimorfonucleares, eosinófilos, linfocitos, algunos macrófagos y células plasmáticas ${ }^{19}$. En nuestro paciente se observó un infiltrado mixto con eosinófilos y presencia de células gigantes como reacción a un cuerpo extraño, mas no se pudo evidenciar atrofia de vellosidades debido a que la biopsia duodenal se realizó de una lesión estenosante friable.

El tratamiento antiparasitario de elección es ivermectina, por su efectividad y buena tolerancia; sin embargo, las dosis óptimas no están definidas en los casos de estrongiloidiasis diseminada/hiperinfección. Las dosis usadas de ivermectina por vía oral o sonda nasogástrica en casos de obstrucción duodenal fueron similares al nuestro, $200 \mu \mathrm{g} / \mathrm{kg}$ diarios , en dos días consecutivos. Sin embargo, en casos de infecciones diseminadas puede repetirse o prolongarse la terapia hasta el aclaramiento microscópico de las larvas en los sitios infectados documentados, sin haber un régimen establecido ${ }^{20}$. El esquema usado con mayor frecuencia $(70 \%)$ en los casos encontrados de obstrucción intestinal estuvo basado en ivermectina como monoterapia o en combinación con albendazol. Cabe destacar que no existen presentaciones parenterales de estos antihelmínticos disponibles para humanos; no obstante, se usan presentaciones veterinarias de ivermectina subcutánea (SC) en casos de estrongiloidosis diseminada que no responden a terapia estándar por vía oral o cuando ésta no está disponible (íleo paralítico o síndrome de mala absorción). Las dosis utilizadas son desde $75 \mu \mathrm{g} / \mathrm{kg}$ a $200 \mu \mathrm{g} / \mathrm{kg}$ diarios o cada $72 \mathrm{~h}^{12,20}$.

Existe evidencia que la coinfección con HTLV-1 puede contribuir a disminuir la eficacia del tratamiento. A su vez promueve la progresión de la infección de este retrovirus en el hospedero, facilitando su replicación con mayor nivel de virus circulante, y que declina luego del tratamiento con ivermectina.

Por último, se ha demostrado que la estrongiloidosis puede ser un co-factor en el desarrollo de leucemia/ linfoma de células T del adulto, acortando el período de latencia de la leucemogénesis ${ }^{17}$; por lo cual es necesario el seguimiento estricto del paciente.

\section{Conclusión}

A pesar de que $S$. stercoralis usualmente causa una infección crónica asintomática u oligosintomática, bajo ciertas condiciones (coinfección con HTLV-1), el número de parásitos pueden incrementarse sustancialmente llevando a una hiperinfección y/o una enfermedad diseminada grave. La obstrucción duodenal es una complicación aparentemente infrecuente, pero potencialmente fatal. La presentación clínica puede ser atípica, haciendo el diagnóstico extremadamente dificultoso. Finalmente, debe considerarse como causa diferencial de obstrucción intestinal alta en países endémicos, como el Perú.

Agradecimientos: Los autores agradecemos a David Huisa Loayza (médico residente de Medicina Interna) y Sandra Velazco (médico residente de Anatomía Patológica) por su colaboración con las fotografías del caso.

\section{Resumen}

La estrongiloidiasis es una enfermedad desatendida en Latinoamérica. Las manifestaciones gastrointestinales son inespecíficas y la obstrucción duodenal es una complicación infrecuente. Presentamos el caso clínico de un varón de 31 años, procedente de la selva central de Perú, que ingresó por una obstrucción intestinal alta, con úlceras y una estenosis duodenal evidenciadas en la endoscopia digestiva alta. El informe histopatológico reveló la presencia de larvas de Strongyloides stercoralis. La evolución clínica y endoscópica fueron favorables con el tratamiento con ivermectina. Existen poco más de 20 casos publicados de obstrucción duodenal por $S$. stercoralis. Adicionalmente, se confirmó una infección por HTLV-1, asociación descrita frecuente.

\section{Referencias bibliográficas}

1.- Greaves D, Coggle S, Pollard C, Aliyu S, Moore E M. Strongyloides stercoralis infection. BMJ 2013; 347: f4610. DOI: 10.1136/bmj. f4610.

2.- Schär F, Trostdorf U, Giardina F, Khieu V, Muth S, Marti H, et al. Strongyloides stercoralis: global distribution and risk factors. PLoS Negl Trop Dis 2013; 7: e2288. DOI: 10.1371/journal.pntd.0002288.
3.- Cruz R J Jr, Vincenzi R, Ketzer B M. Duodenal obstruction - an unusual presentation of Strongyloides stercoralis enteritis: a case report. World J Emerg Surg 2010; 5: 23. DOI: 10.1186/1749-7922-5-23.

4.- Jain V, White S R, Sharma D, Iliev A. Small bowel obstruction from Strongyloides stercoralis: case report. J Clin Case Rep 2014; 4: 346. doi:10.4172/2165-7920.1000346.

5.- Vásquez-Ríos G, Machicado J D, Terashima A, Marcos L A. Irritable bowel syndrome and intestinal parasites: a view from South America. Rev Gastroenterol Peru 2016; 36: 153-8.

6 .- Shields A M, Goderya R, Atta M, Sinha P. Strongyloides stercoralis hyperinfection presenting as subacute small bowel obstruction following immunosuppressive chemotherapy for multiple myeloma. BMJ Case Rep 2014; 2014: bcr2013202234. DOI: 10.1136/bcr-2013202234.

7 .- Figueira C F, Gaspar M T da C, Cos L D, Ussami E Y, Otoch J P, Felipe-Silva A. 
Strongyloides stercoralis hyperinfection associated with impaired intestinal motility disorder. Autops Case Rep 2015; 5: 27-34. DOI: 10.4322/acr.2015.005.

8.- Roseman D, Kabbani D, Kwah J, Bird D, Ingalls R, Gautam A, et al. Strongyloides stercoralis transmission by kidney transplantation in two recipients from a common donor. Am J Transplant 2013;13: 2483-6. DOI: 10.1111/ajt.12390.

9 .- Vithayasai N, Jennuvat S. Gastrointestinal manifestations in severe strongyloidiasis: report of 3 cases and literature review. Southeast Asian J Trop Med Public Health 2010; 41: 227.

10.- Khuroo M S. Hyperinfection strongyloidiasis in renal transplant recipients. BMJ Case Rep 2014; 2014: bcr2014205068. DOI: 10.1136/bcr2014-205068.

11.- Cui R, Dougan S, Leung P, Mclntyre T. Proximal small bowel obstruction and Strongyloides. Int J Surg 2015; 23: S37.

12.- Bogoch I, Khan K, Abrams H, Nott C, Leung E, Fleckenstein L, et al. Failure of ivermectin per rectum to achieve clinically meaningful serum levels in two cases of Strongyloides hyperinfection. Am J Trop Med Hyg 2015; 93: 94-6. DOI: 10.4269/ ajtmh.15-0077.

13.- Barrett J, Broderick C, Soulsby H, Wade P, Newsholme W. Subcutaneous ivermectin use in the treatment of severe Strongyloides stercoralis infection: two case reports and a discussion of the literature. J Antimicrob Chemother 2016; 71: 220-5. DOI: 10.1093/jac/ $\mathrm{dkv} 315$.

14.- Malézieux-Picard A, Saint-Paul M C, Dellamonica J, Courjon J, Tieulié N, Marty $\mathrm{P}$, et al. Severe intestinal obstruction due to Strongyloides stercoralis in a pregnant woman. Med Mal Infect 2017; 47: 429-31. DOI: 10.1016/j.medmal.2017.05.007.

15.- Hindy P, Parvin R, Hanna K, Gress F. Strongyloidiasis presenting as duodenal obstruction in a patient infected with human T-cell lymphotropic virus type 1 . Gastrointest Endosc 2011; 74: 439-41. DOI: 10.1016/j. gie.2010.11.035.

16.- El Hajj W, Nakad G, Abou Rached A. Protein loosing enteropathy secondary to strongyloidiasis: case report and review of the literature. Case Rep Gastrointest Med 2016; 2016: 6831854. DOI: $10.1155 / 2016 / 6831854$.

17.- Weatherhead J, Mejía R. Immune response to infection with Strongyloides stercoralis in patients with infection and hyperinfection. Curr Trop Med Rep 2014; 1: 229-33. https://doi. org/10.1007/s40475-014-0032-9

18.- Requena-Méndez A, Buonfrate D, Bisoffi $Z$, Gutiérrez J M. Advances in the diagnosis of human strongyloidiasis. Curr Trop Med Rep 2014; 1: 207-15. https://doi.org/10.1007/ s40475-014-0034-7.

19.- Arévalo F, Cerrillo G. Strongyloides stercoralis: hallazgos histopatológicos en mucosa duodenal 1999-2005. Rev Gastroenterol Peru 2006; 26: 44-8.

20.- Donadello K, Cristallini S, Taccone F S, Lorent S, Vincent J L, de Backer D, et al. Strongyloides disseminated infection successfully treated with parenteral ivermectin: case report with drug concentration measurements and review of the literature. Int J Antimicrob Agents 2013; 42: 580-3. DOI: $10.1016 / \mathrm{j}$. ijantimicag.2013.07.015. 